\title{
Insufficient Sleep as a Contributor to Weight Gain: An Update
}

\author{
Jean-Philippe Chaput • Angelo Tremblay
}

Published online: 16 August 2012

(C) Springer Science+Business Media, LLC 2012

\begin{abstract}
Behavioral sleep restriction is becoming endemic in modern times. The evidence taken as a whole suggests that insufficient sleep plays a role in the risk of obesity. At present it appears very likely that insufficient sleep results in increased food intake, while there is little support that it results in reduced energy expenditure. New studies provide evidence that insufficient sleep enhances hedonic stimulus processing in the brain underlying the drive to consume food and are consistent with the notion that reduced sleep may lead to greater propensity to overeat. Recent studies also suggest that short sleep duration preferentially increases abdominal adiposity, possibly through a hyperactivation of the hypothalamo-pituitary-adrenal axis. Individuals attempting to lose weight should also consider getting adequate amounts of sleep in addition to limiting calorie intake and increasing physical activity to improve the success of their weight loss intervention. Finally, preliminary results by our research group lend support to the effect that increasing sleeping time in short-duration sleepers has the potential to limit adiposity gain over time. A proof of principle study on a randomized sample is currently under way to assess whether sleep extension is feasible and whether it influences body weight. In summary, the preponderance of the
\end{abstract}

J.-P. Chaput $(\bowtie)$

Healthy Active Living and Obesity Research Group,

Children's Hospital of Eastern Ontario Research Institute,

401 Smyth Road,

Ottawa, Ontario, Canada K1H 8L1

e-mail: jpchaput@cheo.on.ca

\section{A. Tremblay}

Department of Kinesiology, Faculty of Medicine,

Laval University,

2300 de la Terrasse,

Quebec City, Quebec, Canada G1V 0A6

e-mail: angelo.tremblay@kin.ulaval.ca evidence supports taking a pragmatic approach and encouraging a good night's sleep as an adjunct to other health promotion measures.

Keywords Short sleep duration · Sleep deprivation · Energy balance $\cdot$ Body weight $\cdot$ Obesity $\cdot$ Adiposity $\cdot$ Appetite .

Feeding behavior $\cdot$ Physical activity $\cdot$ Exercise $\cdot$ Insufficient sleep · Weight gain

\section{Introduction}

Despite recent data suggesting that the dramatic increases in overweight and obesity seen over the past three decades have begun to plateau, rates remain alarmingly high $[1,2]$. Concurrent with these increases has been a reduction in sleep duration $[3,4]$, raising the question of an association or even that short sleep duration could be a possible cause of obesity $[5,6 \bullet]$. Factors responsible for this secular decline in sleep duration are not well understood, but this deficit has been ascribed to the modern way of living [7]. Interestingly, declines in children's sleep duration have occurred as a result of progressive delays in children's bedtimes, but unchanged wake times [8-10]. Delays in children's bedtimes have been attributed to activities that keep children awake and contexts that allow them to do so such as technology use, schoolwork and part-time employment [11-13]. Similarly, artificial light, caffeine use and parental attitudes have been identified as contexts that allow children to have later bedtimes [14-16].

The importance of having a good night's sleep for body weight stability and overall health is accumulating [17•]. A mounting body of epidemiological evidence associates insufficient sleep not only with obesity $[18,19]$, but also with type 2 diabetes [20, 21], coronary heart disease [22, 23], hypertension [24, 25], and premature death [26, 27]. It thus becomes clearer for researchers that multiple plausible 
causes of obesity exist outside of the conventional risk factors $[28,29]$. Among them, there is robust evidence supporting the role of reduced sleep as contributing factor to the current obesity epidemic [30, 31]. Given that chronic sleep restriction is linked to the modern way of living, the maintenance of a healthy lifestyle appears to be more difficult for short-duration sleepers in the current obesogenic environment.

The objective of this paper is to highlight recent evidence on short sleep duration and its association with obesity. Furthermore, potential mechanisms by which insufficient sleep may predispose to weight gain are discussed.

\section{Association Between Short Sleep Duration and Obesity}

\section{Observational Evidence}

A growing body of epidemiologic evidence associates short sleep duration with obesity and weight gain [6•]. Using a cohort of children aged between 5 and 10 years, we reported that short sleep duration was the most important risk factor for overweight and obesity [32]. Excess body weight was predicted by short sleep duration, with odds ratios exceeding those of other well-known risk factors, including parental obesity, television viewing, socioeconomic status, and physical inactivity. Likewise, we also showed that short sleep duration contributed more importantly to weight gain than other traditional risk factors, such as nonparticipation in high-intensity physical activity and high dietary fat intake in a prospective cohort of adults [33•]. A recent systematic review and meta-analysis by Cappuccio and colleagues reports that children who sleep less than $10 \mathrm{~h}$ per night are at $89 \%$ greater risk of being obese than their peers who sleep more than $10 \mathrm{~h} /$ night [34]. Likewise, adults sleeping $\leq 5 \mathrm{~h}$ per night were $55 \%$ more likely to be obese than those sleeping $>5 \mathrm{~h}$ per night. A pooled regression analysis in adults also showed that a reduction in $1 \mathrm{~h}$ of sleep per day would be associated with a $0.35 \mathrm{~kg} / \mathrm{m}^{2}$ increase in body mass index (BMI) [34]. For a person approximately $178 \mathrm{~cm}$ tall it would be equivalent to approximately $1.4 \mathrm{~kg}$ in weight. Thus, although most studies have found a significant association between inadequate sleep and obesity, the association appears stronger at younger ages, suggesting that children and adolescents may be more vulnerable to the effects of inadequate sleep.

We previously reported results in children showing that the association of sleep duration with waist circumference remained significant even after adjusting for BMI, indicating that short sleep duration is more strongly related to central fat deposition than to overall level of adiposity [35]. We recently corroborated this observation with the use of a 6-year longitudinal design in adults by showing that short sleep duration preferentially increases abdominal adiposity [36•]. This finding is of particular concern because abdominal adiposity is associated with a number of metabolic anomalies. The hyperactivity of the hypothalamopituitary-adrenal (HPA) axis associated with short sleep duration [37] is one possible source of explanation for our findings. Lack of sleep has been reported to constitute a metabolic stressor, with increased cortisol concentrations as the end product [38•]. Because abdominal adipose tissue has more cells per unit of mass, higher blood flow and more glucocorticoid receptors, glucocorticoids could affect abdominal fat to a greater extent than subcutaneous adipose tissue [39]. Of interest, it has been proposed that visceral obesity could represent a physiological adaptation to chronic exposure to stress, particularly when failure to cope is observed [40]. The Hervey's hypothesis which suggests that fat cells take up and catabolize glucocorticoids is one of potential regulatory pathways by which abdominal fat may grow in response to chronic stress exposure [41]. This is also supported by other evidence showing that abdominal obesity is associated with an increased cortisol clearance [42]. Thus, one cannot exclude the possibility that abdominal fat accumulation represents a biological adaptation to chronic lack of sleep.

Although studies examining the relationship between sleep patterns and adiposity status tend to focus on sleep duration, it has recently been reported that sleep timing behavior may be a better predictor of obesity than sleep duration alone. Chronotype studies have shown that morning-type children tend to have a lower BMI than evening-type children $[43,44]$. It is plausible that this may be due to differing opportunities for physical activity and sedentary behaviors in these time slots, with the evening offering relatively few opportunities for physical activity and relatively more opportunities for sedentary behaviors, such as television viewing and video game playing, which have been shown to increase food intake [45]. Results from a recent study involving 2,200 Australian children showed that those who went to bed late and woke up late were 2.2 times more likely to be obese than those who went to bed early and woke up early despite the same amount of sleep [46]. Furthermore, late sleepers were almost twice as likely to have low moderate-to-vigorous intensity physical activity and 2.9 times more likely to sit in front of the TV and computer or play video games for more hours than guidelines recommend. These results agree with those from a recent study in adults showing that late sleepers consumed on average $248 \mathrm{kcal}$ more per day than normal sleepers, with the majority of the excess calories occurring at dinner and after 8:00 PM [47].

With regard to causality in the association between short sleep duration and weight gain using epidemiologic evidence, causal inference is difficult due to lack of control 
for important confounders in many studies (e.g. depression, psychosocial problems, chronic illness, use of hypnotics, etc.) and inconsistent evidence of temporal sequence in prospective studies. Additionally, the findings suggest that there may be bidirectional effects, shortened sleep causing weight gain and weight gain causing shortened sleep, hence creating a setting for a vicious cycle [6•]. Another concern is the rarity of objective measurements of sleep duration (including sleep quality). Although polysomnography is not feasible in large cohorts and the recording instrumentation may itself interfere with sleep, other methods of objectively measuring sleep exist. Wrist actigraphy is definitively one of them and has been validated against polysomnography [48]. Unfortunately, only few studies of sleep duration and obesity to date have utilized actigraphy. The fact that many investigators reported retrospective analysis in this new field of research can explain why many studies have relied on questionnaires to assess sleep. In a population-based sample of middle-aged adults, subjective reports of habitual sleep are moderately correlated with actigraph-measured sleep, but are biased by systematic over-reporting [49]. The wording of these sleep questions varies greatly across studies and few questions have been validated. For example, many studies asked only about nocturnal sleep which may substantially underestimate sleep duration in populations where napping or shift-working is common. Additionally, the large night to night variability in sleep duration may also lead to substantial measurement error. Of particular concern is the fact that sleep habits vary greatly between weekdays and weekends [50]. These differences are even larger in those who have more severe sleep restriction during the week. It is unclear how accurately individuals are able to average their sleep habits over weekdays and weekends to answer a single question about usual sleep habits. Finally, other aspects have the potential to hamper the interpretation of the evidence in terms of causality, such as the body weight history and the possibility of common or upstream underlying causes; however, the preponderance of the evidence taken as a whole points toward an effect of shortened sleep on body weight. Future epidemiologic research from large prospective cohort studies with objective measurement of sleep habits and repeated measures of both sleep duration and adiposity is needed to more definitively establish a causal link and to better define the magnitude of any causal effect.

\section{Experimental Evidence}

Many short-term intervention studies aimed at investigating the effects of sleep restriction on energy balance have been published over the last 10 years and have contributed to our understanding of the potential physiological mechanisms underpinning the short sleep-obesity association. This field of research has certainly been fueled by the apparent paradox that an increase in the time spent in the most sedentary of all activities (i.e., sleep) is associated with leanness. The group of Eve Van Cauter in Chicago has certainly helped to clarify this paradox several years ago, when they experimentally tested the acute effects of sleep restriction on feeding behavior and key appetite-related hormones. The seminal study by Spiegel et al. [51] showed that two consecutive nights of sleep restriction to $4 \mathrm{~h}$ instead of $10 \mathrm{~h}$ induced an $18 \%$ decrease in the circulating concentrations of the anorexigenic hormone leptin and a $28 \%$ increase in the concentrations of the orexigenic hormone ghrelin in conjunction with increased sensations of hunger and appetite. This is concordant with the analysis that we have performed in the Quebec Family Study, which has revealed that short sleepers are characterized by plasma leptin levels that are lower than predicted by their fat mass [52]. Furthermore, these findings concur with some other intervention studies showing that sleep is an important modulator of neuroendocrine function and metabolism. Indeed, sleep restriction has been shown to decrease glucose tolerance, decrease insulin sensitivity, increase evening concentrations of cortisol, increase levels of ghrelin, decrease levels of leptin, and increase the drive to eat [53-56]. However, other intervention studies have not been able to reproduce these findings [57-59], leaving the question open as to whether the connection between short sleep duration and obesity can be explained by changes in homeostatic feeding behavior (i.e., the hormones that trigger hunger and food intake).

An important limitation of experimental studies is that they are short-term, lasting a few weeks at most. This raises the question of whether these effects will persist outside the laboratory when sleep restriction is chronic. Experimental evidence that sleep restriction induces obesity is not possible in humans for both ethical and logistic reasons. The best evidence to show a cause-and-effect association between shortened sleep and weight gain would be to conduct a randomized controlled trial in which we would have to restrict sleep duration in a group of lean people in order to be able to compare them with a control group. However, the slow development of obesity implies that such a study would have to run for years in a large sample of individuals. This means that we need to rely on short-term intervention studies and epidemiologic studies. As recently addressed [6•], short-term experiments may elucidate biological mechanisms supposedly inducing continuous weight gain, but whether they produce obesity in the long run is unknown. Likewise, observations of obesity development in nonobese population groups over time after exposure to short sleep duration can be addressed with the use of various epidemiological methods, each of which have pros and cons. Although the proof-of-concept will never be complete in this field of investigation, the preponderance of the evidence taken as a whole points toward an effect of sleep 
duration on the vulnerability to weight gain. It is also important to remember that a good night's sleep is the "normal" biological condition and no one can effectively argue that lack of sleep is healthy. Therefore, there is minimal risk in taking a pragmatic approach and encouraging a good night's sleep as an adjunct to other health promotion measures $[7,60,61]$.

\section{Potential Mechanisms by Which Short Sleep Duration May Predispose to Weight Gain}

\section{Effects of Short Sleep Duration on Energy Intake}

In order to produce weight gain, reduced sleep must either increase energy intake and/or reduce energy expenditure. The potential mechanisms by which short sleep duration may influence body weight are shown in Fig. 1. The most plausible explanation as to why short sleepers have a higher risk of becoming obese is through an increased food intake. Indeed, many recent intervention studies have reported an increase in food intake after short-term sleep restriction [59, 62-64]. Among the possible explanations, short sleep duration might increase the risk of weight gain by preventing the restoration of a hormonal profile facilitating appetite control (homeostatic drives to eat). As mentioned above, inadequate sleep has been reported in some studies to decrease leptin levels, increase ghrelin level and cortisol levels, alter glucose homeostasis, and activate the orexin system $[65,66]$. However, recent studies have not been able to reproduce these findings and it suggests that the explanation of an upregulation of appetite as a result of sleep restriction is not as robust as previously reported [57-59].

Shortened sleep could also lead to weight gain and obesity by increasing the time available for eating and by making the maintenance of a healthy lifestyle more difficult.
In an environment in which energy-dense food is highly palatable and readily available, caloric intake may be directly proportional to the time spent awake, especially if most of wakefulness is spent in sedentary activities, such as watching television in which snacking is common [45]. This concept is supported by recent data showing that recurrent bedtime restriction under free-living conditions did not down-regulate the satiety hormone leptin nor upregulate the appetite-stimulating hormone ghrelin, but increased intake of calories from snacks, which suggests hedonic rather than homeostatic mediators of the shift in food selection [57]. Results from another study showed that habitual short sleepers (average of $6 \mathrm{~h} / \mathrm{night}$ ) ate more often (i.e., $>3$ meals/ day with more frequent nibbling) than did long sleepers [67]. Likewise, the recent study by Weiss and colleagues [68] provides further evidence to support the theory of reward seeking behaviors associated with short sleep duration. The authors observed that objectively measured shorter sleep duration in adolescents was associated with a relatively higher caloric intake derived from fat and a 2-fold increased risk of consuming $\geq 475 \mathrm{kcal} /$ day from snacks. Accordingly, future studies should put more efforts in investigating the nonhomeostatic, reward-driven eating behavior associated with inadequate sleep [69].

The functional magnetic resonance imaging (fMRI) has recently been instrumental in documenting food-related reward activation in the brain after sleep restriction. Interestingly, a recent study in adolescents reports that impaired sleep was associated with less reactivity of reward-related brain systems, suggesting that more exciting rewards are required to create the same level of neuronal activation [70]. Furthermore, two other recent studies reported that sleep restriction leads to increased activation of brain regions sensitive to food stimuli $[71,72]$. These new studies provide evidence that inadequate sleep enhances hedonic stimulus processing in the brain underlying the drive to
Fig. 1 Potential mechanisms by which insufficient sleep may predispose to weight gain. The symbol (?) denotes a lack of scientific support

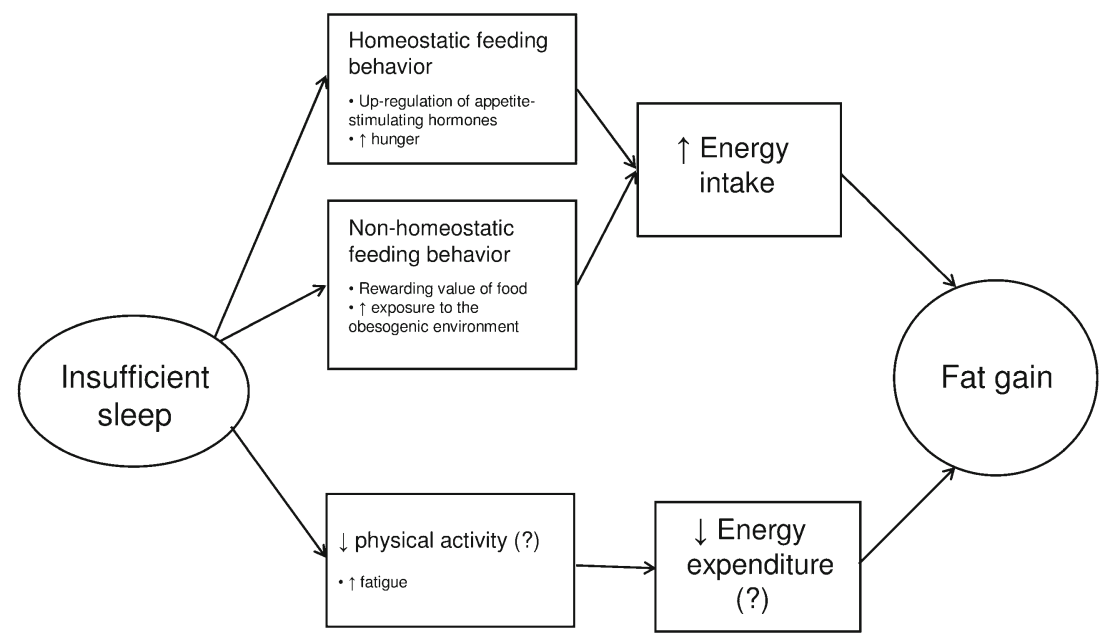


consume food and are consistent with the notion that reduced sleep may lead to greater propensity to overeat.

The fact that studies are not all consistent in showing an effect of shortened sleep on food intake [57, 58] highlights the need for a better characterization of short-duration sleepers. In order to further explain the inter-individual variations in weight gain among short-duration sleepers, we explored this issue by measuring disinhibition eating behavior trait in short- $(\leq 6 \mathrm{~h})$, average- $(7-8 \mathrm{~h})$ and longduration sleepers ( $\geq 9 \mathrm{~h}$ ) over a 6-year follow-up period in adults. We observed that having a high disinhibition eating behavior trait significantly increases the risk of overeating and gaining weight in those with short sleep duration (Fig. 2) [73•]. These novel results emphasize the need to include a disinhibition eating behavior score in the personalized risk assessment of weight gain and obesity of shortduration sleepers. Furthermore, these results suggest that short sleep duration alone may not be sufficient in and of itself to predict weight gain.
Effects of Short Sleep Duration on Energy Expenditure

Decreased energy expenditure represents another potential mechanism to explain the short sleep-obesity connection (Fig. 1). First, the increased fatigue and tiredness associated with sleeping too little could lessen one's resolve to follow physical activity regimens. In particular, recent results showed that short-term sleep restriction (two nights of $4 \mathrm{~h}$ in bed) was accompanied by a decrease in daytime spontaneous physical activity in healthy men [58]. Interestingly, the reduction in overall physical activity observed was a shift toward less intense activities under free-living conditions. Thus, these findings suggest that reduced voluntary physical activity could be another mechanism linking insufficient sleep to weight gain. However, we recently reviewed the evidence on short sleep duration and its association with energy expenditure and on the basis of the available literature, we concluded that short sleep duration does not seem to substantially affect total energy expenditure, nor is there
Fig. 2 (a) Six-year weight gain and (b) energy intake according to sleep duration group and disinhibition eating behavior trait (high vs. low). Values are mean \pm SEM. LDEB: low disinhibition eating behavior trait tertile (score $\leq 3$ ). HDEB: high disinhibition eating behavior trait tertile (score $\geq 6$ ). Short sleepers $(\leq 6 \mathrm{~h} /$ night $)$, average sleepers (7-8 h/night) and long sleepers ( $\geq 9 \mathrm{~h} /$ night). Statistical significance was assessed by analysis of covariance, followed by a Tukey HSD post-hoc test. The model was adjusted for age, sex, baseline body weight, length of followup, employment status, highest educational level, total annual family income, menopausal status, shift-working history, and alcohol intake as covariates. *Significantly different from all other groups $(P<0.05)$. Figure adapted from Chaput et al. [73•]
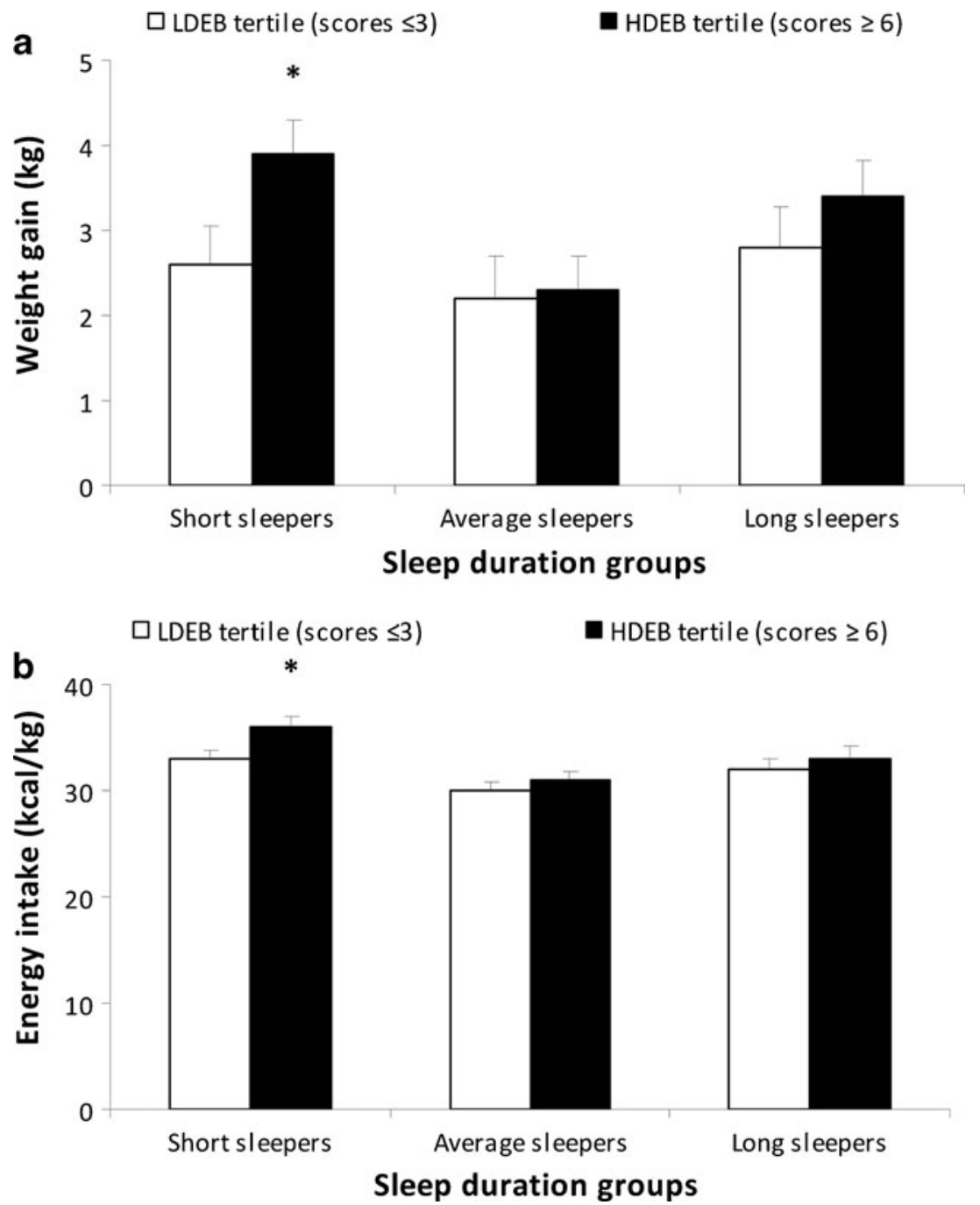
sufficient evidence in support of any meaningful effect of restricted sleep on the components of energy expenditure [74•]. A brief overview of the effects of short sleep duration on the components of energy expenditure in humans is addressed in the next sections.

\section{Total Energy Expenditure (TEE)}

Since sleeping metabolic rate is lower than basal metabolic rate, the difference in the extra time awake between normal sleepers and short sleepers can potentially induce a difference in TEE, even if the extra time spent awake is very sedentary. Thus, it can be hypothesized that any difference in TEE between short sleep and long sleep conditions is influenced by the time awake alone and not necessarily ascribed to a change in global mechanisms involved in the regulation of energy metabolism, apart from the effect of arousal and physical activity. In their cross-over study, Nedeltcheva et al. [57] used the doubly-labeled water technique to measure the difference in TEE after 14 days of $5.5 \mathrm{~h}$ and $8.5 \mathrm{~h}$ of sleep in overweight adults and they reported no change in TEE between the two sleep conditions. Similar results were obtained by St-Onge et al. [62] who reported that TEE did not change with sleep restriction as measured with the doubly-labeled water method. Likewise, a recent study by Hursel et al. [75] reported no difference in $48 \mathrm{~h}$ TEE measured in a respiration chamber in healthy men. In this study, the authors used a twocondition, crossover study over two consecutive nights where time in bed was equal in the two conditions (23:007:30) but with fragmented sleep in one condition. Another study by Jung et al. [76] found a $7 \%$ increase in TEE measured in a respiration chamber after $40 \mathrm{~h}$ of total sleep deprivation. However, the difference in energy expenditure only persisted during habitual sleep time; hence, the increased $24 \mathrm{~h}$ energy expenditure corresponded to the energy demand of prolonged wakefulness and cannot be ascribed to an intrinsic change in metabolism as a consequence of sleep restriction. Along the same lines, Bosy-Westphal et al. [59] did not find any difference in TEE compared to baseline in a controlled laboratory setting including women subjected to four consecutive nights of increasing sleep deficit. Collectively, the experimental evidence in humans does not suggest that sleep restriction affects TEE.

\section{Resting Metabolic Rate (RMR)}

A small number of studies have examined RMR after an experimentally-induced sleep deficit. In most cases, sleep restriction did not change RMR [57, 59, 62, 75]. In contrast to these findings, a recent study by Benedict et al. [77] measuring RMR after one night of total sleep deprivation in a group of 14 normal-weight men showed a significant decrease in RMR. However, the external generalizability of these findings is limited due to the fact that total sleep deprivation (extreme situation) is not common in a real-life setting. Furthermore, it is well known that slow-wave sleep has an essential energy preserving function. Given that slow-wave sleep has been shown to be preserved in partial sleep deprivation conditions [38•], this can explain the discrepancy between partial and total sleep deprivation with regard to RMR. We also used indirect calorimetry in our 6year prospective cohort study and found no difference in RMR between short- $(\leq 6 \mathrm{~h})$, average- $(7-8 \mathrm{~h})$ and longduration sleepers $(\geq 9$ h) [18]. Taken together, sleep restriction does not seem to significantly impact RMR in the shortterm but the long-term effects are still unknown. Because even small undetectable and statistically insignificant changes in RMR of about 1-2 \% can over a life span make a difference in term of weight control, it is possible that the methods used to measure RMR are not accurate enough to capture such small changes.

\section{Physical Activity Energy Expenditure (PAEE)}

PAEE is the most variable of the TEE components and plays an important role in the regulation of energy balance. Hypothetically, insufficient sleep can influence physical activity in a number of ways. On a behavioral level, shortened sleep may cause people to be less active as a consequence of increased tiredness. On the other hand, it cannot be ruled out that tiredness can cause people to be more active in order to combat this state of fatigue, basically keeping awake by moving. Additionally, PAEE should theoretically increase for every additional hour spent awake compared to sleeping (at least for light-intensity physical activity). At present, at least four experimental studies have directly examined the acute effect of sleep restriction on physical activity. Using accelerometry in a randomized crossover study, Brondel et al. [64] observed that physical activity in free-living conditions was significantly higher after $4 \mathrm{~h}$ of sleep than after $8 \mathrm{~h}$ of sleep, even though the sensation of sleepiness was more marked. This is in contrast with the crossover study by Schmid et al. [58] who found a significantly lower spontaneous physical activity measured with accelerometry after sleep restriction and a significant shift in the intensity of activity toward low-level physical activity in the short sleep condition. Using step counts in a free-living condition, Bosy-Westphal et al. [59] did not find any difference in physical activity before and after four nights of increasing sleep deficit. Finally, Hursel et al. [75] reported a $7 \%$ increase in physical activity in the sleep restriction condition, but the strength of this finding is compromised by the fact that the subjects were confined in a calorimetric chamber and were severely limited in their movements. Similarly, observational studies examining the association between 
short sleep duration and physical activity are also conflicting and no clear conclusion can be drawn. Most of them are limited by the fact that both sleep time and physical activity were recorded using questionnaires or interviews. Overall, the evidence on the effects of insufficient sleep on PAEE is unclear and limited. The large inter-individual variation in physical activity level and pattern suggests that any change in physical activity as a result of short sleep duration is probably more related to individual, behavioral aspects than intrinsic, physiological alterations.

\section{Diet-Induced Thermogenesis (DIT)}

Although DIT normally only comprises about $10 \%$ of TEE when subjects are in energy balance, changes in DIT can offer another physiological explanation for the influence of insufficient sleep on energy balance. At present, at least four studies have investigated variations in this component of TEE. Bosy-Westphal et al. [59] investigated the effect of sleep restriction on energy expenditure over $180 \mathrm{~min}$ after an oral glucose load of $1 \mathrm{~kg} / \mathrm{kg}$ of body weight. The authors reported that the glucose-induced thermogenesis (GIT) increased by $34 \%$ after sleep restriction without changes in either postprandial glucose or insulin levels. This increase was calculated on the basis of area calculations of the GIT curves during the glucose load, but it is unclear whether this is the sole effect of the glucose load or whether it also includes RMR. If the latter is true, the relative increase in GIT is, in fact, underestimated. However, since approximately half of the subjects had a close to zero or even a decrease GIT after sleep restriction, the relative increase in GIT reported did not translate into a significantly increased EE during this time period. Furthermore, the rather large increase reported by the authors covers a large interindividual variation and a dependency on initial body fat. In contrast to this finding, Benedict et al. [77] reported a transient decrease in DIT of about $20 \%$ measured over $4 \mathrm{~h}$ after ingesting a liquid formula in the morning after one night of total sleep deprivation. Moreover, neither of the two studies by Nedeltcheva et al. [57, 78•] found any changes in DIT during the $4 \mathrm{~h}$ following a standardized meal challenge. From the limited amount of data, which also differ in their results and methodology, it remains inconclusive whether sleep restriction has a significant impact on DIT.

\section{Substrate Utilization}

Another aspect of energy metabolism is substrate utilization and partitioning; however, there is not much support in the literature for a change in substrate utilization as a result of sleep restriction. Jung et al. [76] measured $24 \mathrm{~h}$ respiratory quotient (RQ) using a whole-body indirect calorimeter both during sleep and during wakefulness and found no difference in either conditions. Likewise, Bosy-Westphal et al. [59] found no change in fasting RQ using a ventilated hood system. In contrast, Nedeltcheva et al. [78•] reported an increase in both fasting RQ and postprandial RQ in the sleep restriction condition following an energy-restricted diet and Hursel et al. [75] also reported an increase in fasting RQ. However, the sleep protocols in these two studies are very different (14 nights of partial sleep deprivation compared to one night of total sleep deprivation) and cannot be compared. Taken together, it is premature to conclude on whether substrate utilization is affected by sleep restriction.

\section{Adaptive Thermogenesis}

Sleep restriction has been demonstrated to influence adaptive thermogenesis in rodent models via an elevated expression of uncoupling proteins, which regulate metabolic rate through thermogenesis $[79,80]$. However, the influence of sleeping habits on adaptive thermogenesis (i.e., greater than expected decreases in resting energy expenditure in response to weight loss) is largely unknown in humans. Of interest, Nedeltcheva et al. [78 $]$ recently reported that resting energy expenditure was significantly lower at the end of a 5.5 -h vs. 8.5 -h time-inbed condition in a laboratory setting and this decrease in resting energy expenditure was greater than expected on the basis of the observed loss of fat and fat-free body mass alone. However, we recently reported results from a naturalistic study showing that both total sleep time and sleep quality are not predictive of a greater than predicted decrease in resting energy expenditure after body weight loss in adults subjected to small caloric restriction $(-300 \mathrm{kcal} /$ day on average) [81]. Future studies should thus put efforts in determining whether sleeping habits impact adaptive thermogenesis in individuals subjected to more severe dietary restriction.

\section{Does Sleep Extension Influence Body Weight Regulation?}

Trials assessing the effects of sleep prolongation on weight change and appetite control among obese individuals suffering from sleep deprivation are needed to evaluate the net benefits of prolonging sleep. A prospective randomized controlled trial is currently under way in the USA in order to measure the effects of an increase in sleep length on body weight in obese short-duration sleepers [82 •]. Nonpharmacological and behavior-based interventions are used to increase sleep duration to a healthy length (approximately $7.5 \mathrm{~h}$ ) in obese adults sleeping less than $6.5 \mathrm{~h}$ per night. Preliminary observations from this proof-of-concept study were published in 2010 and showed that the participants in the intervention group who have increased sleep duration reported a better mood and ability to focus, a decrease in 
sleepiness during the day, more willingness to exercise, as well as a decrease in caffeine intake and less cravings for sweet and salty foods during the evening. These preliminary observations thus suggest that extending sleep duration could have beneficial effects on both mental and physical health in obese short-duration sleepers. It is also important to target the root causes of voluntary sleep restriction; the reasons can be very different between individuals.

Along the same lines, we recently investigated whether a favorable change in sleep duration over 6 years could impact objective indicators of adiposity in adults with the use of a longitudinal, observational study design [83•]. This study was the first to test whether a spontaneous change in sleep duration (from $\leq 6 \mathrm{~h}$ per day to $7-8 \mathrm{~h}$ per day) could influence adiposity. We observed that shifting sleep duration from a short to a healthier length is associated with an attenuation of fat mass gain over time (Fig. 3). This observation is of high clinical relevance and justifies the inclusion of sleep duration in the list of environmental factors that contribute to weight gain.

\section{Does Sleeping Habits Affect the Success of a Weight-Loss Intervention?}

Energy restriction is widely used by obese individuals as a weight loss strategy in order to improve their metabolic profile. However, prescribing dietary interventions to obese,

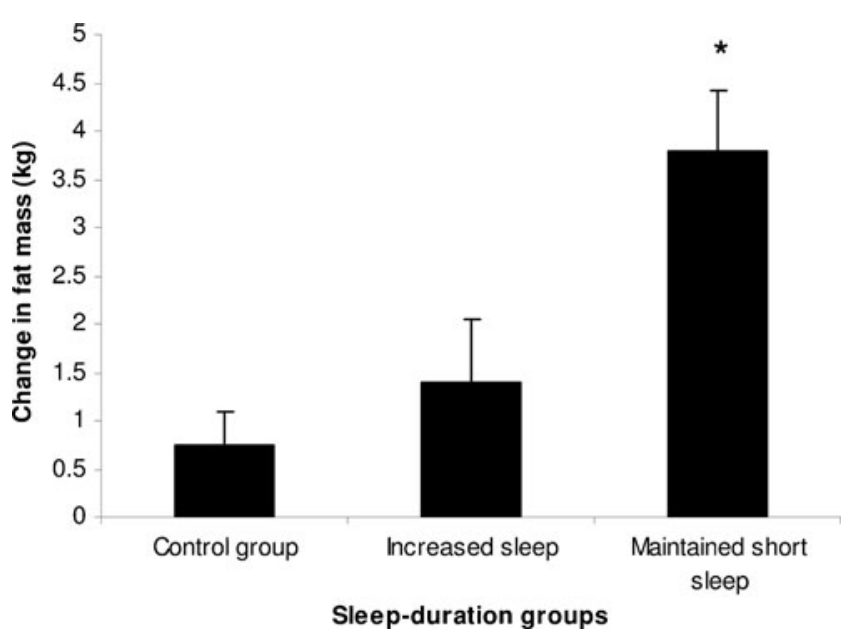

Fig. 3 Mean change in fat mass by sleep-duration group. Values are mean \pm SEM. Control group: individuals who reported sleeping $7-8 \mathrm{~h}$ per day at both baseline and year 6 . Short-duration sleepers who increased their sleep duration: from short sleep duration $(\leq 6 \mathrm{~h}$ per day) at baseline to a healthier length of $7-8 \mathrm{~h}$ per day at year 6 . Short-duration sleepers who maintained their short sleep duration: $\leq 6 \mathrm{~h}$ per day at both baseline and year 6 . Statistical significance was assessed by analysis of covariance, followed by a Tukey HSD post-hoc test. The model was adjusted for age, sex, baseline body mass index, smoking habits, employment status, highest educational level, total annual family income, menopausal status, energy intake and moderate-to-vigorous physical activity as covariates. ${ }^{*} P<0.05$ versus two other groups. Figure adapted from Chaput et al. [83•] short-duration sleepers might be counterproductive as most of them already have higher hunger levels [54]. Moreover, lack of sleep has been reported in some studies to decrease plasma leptin levels, increase plasma ghrelin and cortisol levels, alter glucose homeostasis, and activate the orexin system, all of which impact the control of appetite and might compromise the efficacy of dietary interventions [38•]. Interestingly, Nedeltcheva et al. [78•] recently reported that insufficient sleep undermines dietary efforts to reduce adiposity. In their crossover study, ten overweight adults were randomly assigned to sleep either $5.5 \mathrm{~h}$ or $8.5 \mathrm{~h}$ each night for 14 days in conjunction with moderate caloric restriction in a closed clinical research environment. Compared with participants who slept $8.5 \mathrm{~h}$ per night, participants who slept only $5.5 \mathrm{~h}$ lost less body fat and more fat-free body mass, and had less favorable changes in metabolic hormones and in substrate and energy utilization. In addition, participants in the shorter sleep group perceived greater hunger than participants in the longer sleep group. Given that higher ghrelin concentrations may facilitate the retention of fat [84, 85], and increased hunger could compromise adherence to caloric restriction, these mechanisms may help explain why lack of sufficient sleep may compromise the efficacy of commonly used dietary interventions.

Although these findings are novel and suggest that adequate sleep might be an important factor in successful weight loss, these results need to be replicated in a real life setting because the tightly controlled sleep laboratory setting does not provide information about other environmental factors that might influence compliance to weight-reducing diets. In order to answer this question, we recently verified whether sleeping habits affect the magnitude of fat loss in overweight and obese adults subjected to moderate caloric restriction. We observed that both total sleep time and sleep quality at baseline predicted fat mass loss during typical dietary interventions in overweight and obese adults [86 ${ }^{\circ}$. Specifically, an increase by $1 \mathrm{~h}$ in sleep duration was associated with a decrease of $0.7 \mathrm{~kg}$ in fat mass after adjustment for covariates (Fig. 4). Our results agree with a recent study showing that sleep time predicted success in a weight loss program consisting of an intensive 6-month behavioral weight loss intervention in 472 obese adult participants [87]. The intervention consisted of 22 group sessions led by behavioral counselors and recommendations included reducing dietary intake by $500 \mathrm{kcal} / \mathrm{day}$, adopting the Dietary Approaches to Stop Hypertension (DASH) dietary pattern and increasing physical activity to at least $180 \mathrm{~min}$ per week. Specifically, the authors found that participants sleeping $\leq 6 \mathrm{~h}$ or $\geq 8 \mathrm{~h}$ daily were less likely to achieve eligibility for Phase II (i.e., the randomized weight loss maintenance portion of the study) than those sleeping $>6$ and $\leq 8 \mathrm{~h}$ a day. Thus, these observations further emphasize the need to consider "sleep" as part of any weight loss intervention. 
Fig. 4 Sleep duration in relation to body fat loss in adults. The model was adjusted for age, sex, baseline body mass index, length of the intervention and the change in energy intake as covariates. Figure adapted from Chaput et al. [86•]

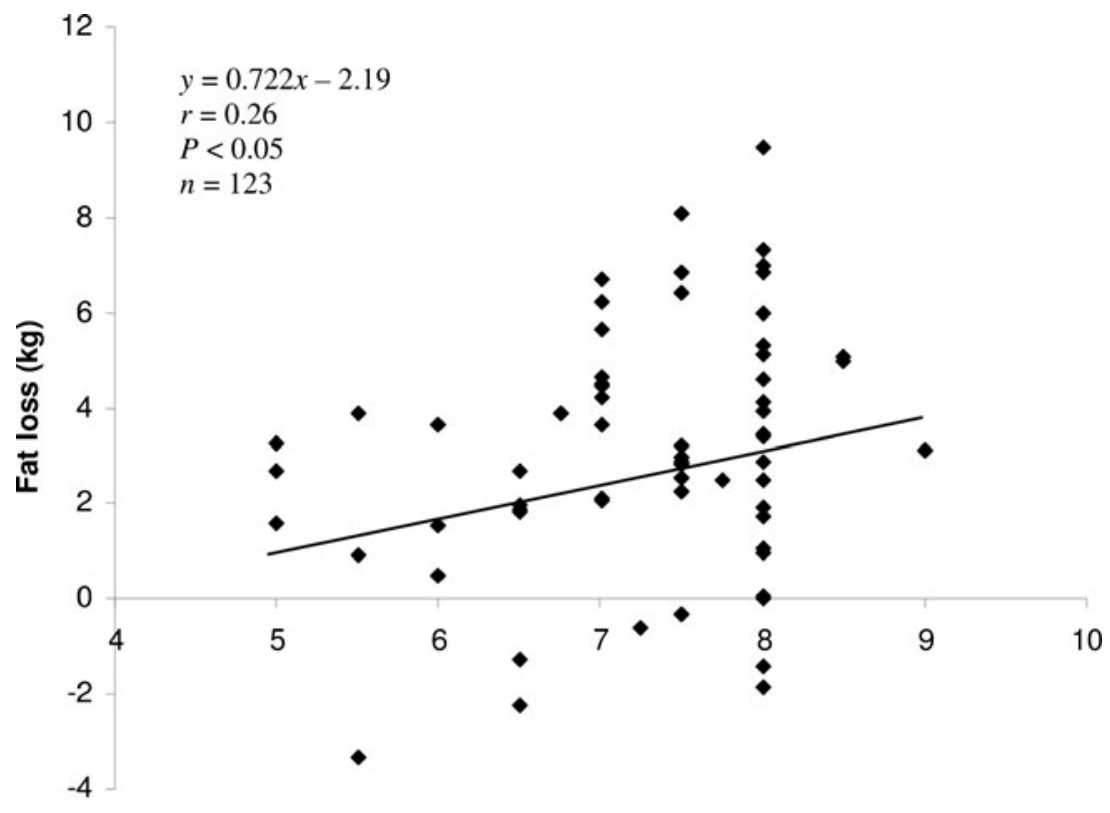

Sleep duration (h)

\section{Insufficient Sleep and Weight Gain: What is the Contribution of the Stress System?}

The two main components of the stress system are the HPA axis and the sympathetic nervous system. The stress responses have been advantageous during evolution to insure survival in environments with limited food supply and marked seasonality [88]. However, contemporary environments have predictable and unlimited food availability, an attenuated seasonality due to artificial lighting, indoor heating during the winter and air conditioning during the summer, and promote sedentariness and overeating. Modern lifestyle stress generally occurs in a sleep-deprived, sedentary, energy abundant environment, which predominantly activates the HPA axis and induces cortisol release and enhances visceral fat accumulation [89].

Experimentally-induced sleep restriction has been shown to increase evening cortisol levels [37]. Even if modest, this elevation in cortisolemia may result, under conditions of chronic sleep loss, in a significant glucocorticoid overload and consequently expose the body to the central and peripheral deleterious effects associated with glucocorticoid excess. In this regard, it is notable that chronic short sleepers have been found to have higher nocturnal cortisol levels compared with chronic long sleepers [90], suggesting that, even in the long term, mechanisms of adaptation and downregulation of the HPA axis fail to occur. In a cross-sectional analysis of data collected from the Whitehall II study, the relationship between self-reported sleep duration, sleep disturbances, and salivary cortisol levels has been assessed in a cohort of more than 2,700 middle-aged men and women [91]. Short sleep duration was associated with an acute increased cortisol awakening response and both short sleep duration and disturbances were independently associated with a slower rate of decline of cortisol levels across the day and thus with increased evening levels.

As mentioned earlier in the paper, we recently published results to the effect that short sleep duration preferentially increases abdominal adiposity rather than overall adiposity [35, 36 $]$. Because stress-induced HPA axis activation has been shown to play a role in body fat accumulation in the abdominal region [39, 40], the HPA axis hyperactivity associated with short sleep duration could be an important mechanism involved. Given that insufficient sleep predominantly activates the HPA axis over the sympathetic nervous system in the modern environment, visceral fat accumulation might become a normal consequence of such a lifestyle habit.

\section{Conclusions}

At present it appears very likely that insufficient sleep results in increased food intake, while there is little evidence that it results in reduced energy expenditure. When these biological mechanisms are considered alongside the consistent relationship between shortened sleep and obesity in prospective studies, and secular trends in sleep duration, there is currently strong evidence that shortened sleep plays a role in the obesity epidemic. While future research will contribute to elucidate other questions in this field of research, a good night's sleep should, in the meantime, be promoted for the maintenance of overall health. 
Acknowledgments Jean-Philippe Chaput holds a Junior Research Chair in Healthy Active Living and Obesity Research. Angelo Tremblay is partly funded by the Canada Research Chair in Environment and Energy Balance.

Disclosure No potential conflicts of interest relevant to this article were reported.

\section{References}

Papers of particular interest, published recently, have been highlighted as:

- Of importance

1. Olds T, Maher C, Zumin S, et al. Evidence that the prevalence of childhood overweight is plateauing: data from nine countries. Int J Pediatr Obes. 2011;6:342-60.

2. Rokholm B, Baker JL, Sørensen TI. The levelling off of the obesity epidemic since the year 1999: a review of evidence and perspectives. Obes Rev. 2010;11:835-46.

3. Matricciani L, Olds T, Petkov J. In search of lost sleep: Secular trends in the sleep time of school-aged children and adolescents. Sleep Med Rev. 2012;16:203-11.

4. National Sleep Foundation. Sleep in America poll. Washington DC: National Sleep Foundation, 2002. Available at http:// www.sleepfoundation.org/article/sleep-america-polls/2002adult-sleep-habits. Accessed April 2012.

5. Chaput JP. Short sleep duration as a cause of obesity: myth or reality? Obes Rev. 2011;12:e2-3.

6. - Nielsen LS, Danielsen KV, Sørensen TI. Short sleep duration as a possible cause of obesity: critical analysis of the epidemiological evidence. Obes Rev. 2011;12:78-92. This paper systematically searched the literature for epidemiological evidence for an association of short sleep duration with weight gain and the authors have analyzed the conceptual and methodological aspects of the evidence.

7. Chaput JP. A good night's sleep for a healthier population. Am J Prev Med. 2010;38:349.

8. Dollman J, Ridley K, Olds T, Lowe E. Trends in the duration of school-day sleep among 10- to 15-year-old South Australians between 1985 and 2004. Acta Paediatr. 2007;96: 1011-4.

9. Iglowstein I, Jenni O, Molinari L, Largo R. Sleep duration from infancy to adolescence: reference values and generational trends. Pediatrics. 2003;111:302-7.

10. Thorleifsdottir B, Björnsson JK, Benediktsdottir B, et al. Sleep and sleep habits from childhood to young adulthood over a 10 -year period. J Psychosom Res. 2002;53:529-37.

11. Van den Bulck J. The effects of media on sleep. Adolesc Med State Art Rev. 2010;21:418-29.

12. Van den Bulck J. Television viewing, computer game playing, and Internet use and self-reported time to bed and time out of bed in secondary-school children. Sleep. 2004;27:101-4.

13. Carskadon MA. Sleep in adolescents: the perfect storm. Pediatr Clin North Am. 2011;58:637-47.

14. Gangwisch JE. Epidemiological evidence for the links between sleep, circadian rhythms and metabolism. Obes Rev. 2009;10 Suppl 2:37-45.

15. Calamaro C, Mason T, Ratcliffe S. Adolescents living the $24 / 7$ lifestyle: effects of caffeine and technology on sleep duration and daytime functioning. Pediatrics. 2009;123:1005-10.

16. de Sousa I, Araućjo J, De Azevedo C. The effect of a sleep hygiene education program on the sleep-wake cycle of Brazilian adolescent students. Sleep Biol Rhythms. 2007;5:251-8.
17. • Chaput JP, Klingenberg L, Sjödin A. Do all sedentary activities lead to weight gain: sleep does not. Curr Opin Clin Nutr Metab Care. 2010;13:601-7. This paper discussed the benefits of having $a$ good night's sleep for body weight stability.

18. Chaput JP, Després JP, Bouchard C, Tremblay A. The association between sleep duration and weight gain in adults: a 6-year prospective study from the Quebec Family Study. Sleep. 2008;31:517-23.

19. Patel SR, Hu FB. Short sleep duration and weight gain: a systematic review. Obesity (Silver Spring). 2008;16:643-53.

20. Cappuccio FP, D'Elia L, Strazzullo P, Miller MA. Quantity and quality of sleep and incidence of type 2 diabetes: a systematic review and meta-analysis. Diabetes Care. 2010;33:414-20.

21. Chaput JP, Després JP, Bouchard C, et al. Sleep duration as a risk factor for the development of type 2 diabetes or impaired glucose tolerance: analyses of the Quebec Family Study. Sleep Med. 2009;10:919-24

22. Cappuccio FP, Cooper D, D'Elia L, et al. Sleep duration predicts cardiovascular outcomes: a systematic review and meta-analysis of prospective studies. Eur Heart J. 2011;32:1484-92.

23. King CR, Knutson KL, Rathouz PJ, et al. Short sleep duration and incident coronary artery calcification. JAMA. 2008;300:2859-66.

24. Knutson KL, Van Cauter E, Rathouz P, et al. Association between sleep and blood pressure in midlife: the CARDIA Sleep Study. Arch Intern Med. 2009;169:1055-61.

25. Gangwisch JE, Heymsfield SB, Boden-Albala B, et al. Short sleep as a risk factor for hypertension: analyses of the first National Health and Nutrition Examination Survey. Hypertension. 2006; 47:833-9.

26. Cappuccio FP, D'Elia L, Strazzullo P, Miller MA. Sleep duration and all-cause mortality: a systematic review and meta-analysis of prospective studies. Sleep. 2010;33:585-92.

27. Gallicchio L, Kalesan B. Sleep duration and mortality: a systematic review and meta-analysis. J Sleep Res. 2009;18:148-58.

28. McAllister EJ, Dhurandhar NV, Keith SW, et al. Ten putative contributors to the obesity epidemic. Crit Rev Food Sci Nutr. 2009;49:868-913.

29. Tremblay A, Chaput JP. About unsuspected potential determinants of obesity. Appl Physiol Nutr Metab. 2008;33:791-6.

30. Saunders T. Potential contributors to the Canadian pediatric obesity epidemic. ISRN Pediatrics. 2011;2011:1-11.

31. Patel SR. Reduced sleep as an obesity risk factor. Obes Rev. 2009;10 Suppl 2:61-8.

32. Chaput JP, Brunet M, Tremblay A. Relationship between short sleeping hours and childhood overweight/obesity: results from the "Québec en Forme" Project. Int J Obes. 2006;30:1080-5.

33. - Chaput JP, Leblanc C, Pérusse L, et al. Risk factors for adult overweight and obesity in the Quebec Family Study: have we been barking up the wrong tree? Obesity (Silver Spring). 2009;17:196470. This study found that short sleep duration was the most important predictor of weight gain and obesity development in this cohort of adults.

34. Cappuccio FP, Taggart FM, Kandala NB, et al. Meta-analysis of short sleep duration and obesity in children and adults. Sleep. 2008;31:619-26.

35. Chaput JP, Tremblay A. Does short sleep duration favor abdominal adiposity in children? Int J Pediatr Obes. 2007;2:188-91.

36. - Chaput JP, Després JP, Bouchard C, Tremblay A. Short sleep duration preferentially increases abdominal adiposity in adults: preliminary evidence. Clin Obes. 2011;1:141-6. This was the first study to report that short sleep duration is associated with a preferential increase in abdominal adiposity in adults.

37. Balbo M, Leproult R, Van Cauter E. Impact of sleep and its disturbances on hypothalamo-pituitary-adrenal axis activity. Int $\mathrm{J}$ Endocrinol. 2010;2010:1-16. 
38. - Leproult R, Van Cauter E. Role of sleep and sleep loss in hormonal release and metabolism. Endocr Dev. 2010;17:11-21. This review paper discusses the contribution of sleep in neuroendocrine function and metabolism.

39. Björntorp P. Do stress reactions cause abdominal obesity and comorbidities? Obes Rev. 2001;2:73-86.

40. Drapeau V, Therrien F, Richard D, Tremblay A. Is visceral obesity a physiological adaptation to stress? Panminerva Med. 2003;45:189-95.

41. Cabanac M, Richard D. The nature of the ponderostat: Hervey's hypothesis revived. Appetite. 1996;26:45-54.

42. Lottenberg SA, Giannella-Neto D, Derendorf H, et al. Effect of fat distribution on the pharmacokinetics of cortisol in obesity. Int $\mathbf{J}$ Clin Pharmacol Ther. 1998;36:501-5.

43. Gaina A, Sekine M, Kanayama H, et al. Morning-evening preference: sleep pattern spectrum and lifestyle habits among Japanese junior high school pupils. Chronobiol Int. 2006;23:607-21.

44. Schubert E, Randler C. Association between chronotype and the constructs of the three-factor eating questionnaire. Appetite. 2008;51:501-5.

45. Chaput JP, Klingenberg L, Astrup A, Sjödin AM. Modern sedentary activities promote overconsumption of food in our current obesogenic environment. Obes Rev. 2011;12:e12-20.

46. Olds TS, Maher CA, Matricciani L. Sleep duration or bedtime? Exploring the relationship between sleep habits and weight status and activity patterns. Sleep. 2011;34:1299-307.

47. Baron KG, Reid KJ, Kern AS, Zee PC. Role of sleep timing in caloric intake and BMI. Obesity (Silver Spring). 2011;19:1374-81.

48. Hyde M, O'Driscoll DM, Binette S, et al. Validation of actigraphy for determining sleep and wake in children with sleep disordered breathing. J Sleep Res. 2007;16:213-6.

49. Lauderdale DS, Knutson KL, Yan LL, et al. Self-reported and measured sleep duration: how similar are they? Epidemiology. 2008; $19: 838-45$.

50. Lauderdale DS, Knutson KL, Yan LL, et al. Objectively measured sleep characteristics among early-middle-aged adults: the CARDIA study. Am J Epidemiol. 2006;164:5-16.

51. Spiegel K, Tasali E, Penev P, Van Cauter E. Brief communication: sleep curtailment in healthy young men is associated with decreased leptin levels, elevated ghrelin levels, and increased hunger and appetite. Ann Intern Med. 2004;141:846-50.

52. Chaput JP, Després JP, Bouchard C, Tremblay A. Short sleep duration is associated with reduced leptin levels and increased adiposity: results from the Quebec Family Study. Obesity (Silver Spring). 2007;15:253-61.

53. Omisade A, Buxton OM, Rusak B. Impact of acute sleep restriction on cortisol and leptin levels in young women. Physiol Behav. 2010;99:651-6.

54. Spiegel K, Tasali E, Leproult R, Van Cauter E. Effects of poor and short sleep on glucose metabolism and obesity risk. Nat Rev Endocrinol. 2009;5:253-61.

55. Magee CA, Huang XF, Iverson DC, Caputi P. Acute sleep restriction alters neuroendocrine hormones and appetite in healthy male adults. Sleep Biol Rhythms. 2009;7:125-7.

56. Schmid SM, Hallschmid M, Jauch-Chara K, et al. A single night of sleep deprivation increases ghrelin levels and feelings of hunger in normal-weight healthy men. J Sleep Res. 2008;17:331-4.

57. Nedeltcheva AV, Kilkus JM, Imperial J, et al. Sleep curtailment is accompanied by increased intake of calories from snacks. Am J Clin Nutr. 2009;89:126-33.

58. Schmid SM, Hallschmid M, Jauch-Chara K, et al. Short-term sleep loss decreases physical activity under free-living conditions but does not increase food intake under time-deprived laboratory conditions in healthy men. Am J Clin Nutr. 2009;90:1476-82.

59. Bosy-Westphal A, Hinrichs S, Jauch-Chara K, et al. Influence of partial sleep deprivation on energy balance and insulin sensitivity in healthy women. Obes Facts. 2008;1:266-73.
60. Ayas NT. If you weight too much, maybe you should try sleeping more. Sleep. 2010;33:143-4.

61. Young T. Increasing sleep duration for a healthier (and less obese?) population tomorrow. Sleep. 2008;31:593-4.

62. St-Onge MP, Roberts AL, Chen J, et al. Short sleep duration increases energy intakes but does not change energy expenditure in normal-weight individuals. Am J Clin Nutr. 2011;94:410-6.

63. Heath G, Roach GD, Dorrian J, et al. The effect of sleep restriction on snacking behaviour during a week of simulated shiftwork. Accid Anal Prev. 2012;45:62-7.

64. Brondel L, Romer MA, Nougues PM, Touyarou P, Davenne D. Acute partial sleep deprivation increases food intake in healthy men. Am J Clin Nutr. 2010;91:1550-9.

65. Knutson K. Does inadequate sleep play a role in vulnerability to obesity? Am J Hum Biol. 2012;24:361-71.

66. Morselli LL, Guyon A, Spiegel K. Sleep and metabolic function. Pflugers Arch. 2012;463:139-60.

67. Hicks RA, McTighe S, Juarez M. Sleep duration and eating behaviors of college students. Percept Mot Skills. 1986;62:25-6.

68. Weiss A, Xu F, Storfer-Isser A, et al. The association of sleep duration with adolescents' fat and carbohydrate consumption. Sleep. 2010;33:1201-9.

69. Chaput JP. Short sleep duration promoting overconsumption of food: a reward-driven eating behavior? Sleep. 2010;33:1135-6.

70. Holm SM, Forbes EE, Ryan ND, et al. Reward-related brain function and sleep in pre/early pubertal and mid/late pubertal adolescents. J Adolesc Health. 2009;45:326-34.

71. Benedict C, Brooks SJ, O'Daly OG, et al. Acute sleep deprivation enhances the brain's response to hedonic food stimuli: an fMRI study. J Clin Endocrinol Metab. 2012;97:E443-7.

72. St-Onge MP, McReynolds A, Trivedi ZB, et al. Sleep restriction leads to increased activation of brain regions sensitive to food stimuli. Am J Clin Nutr. 2012;95:818-24.

73. • Chaput JP, Després JP, Bouchard C, Tremblay A. The association between sleep duration and weight gain is dependent on disinhibited eating behavior in adults. Sleep. 2011;34:1291-7. This study showed that having a high disinhibition eating behavior trait significantly increases the risk of overeating and gaining weight in adults characterized by short sleep duration.

74. • Klingenberg L, Sjödin A, Holmbäck U, et al. Short sleep duration and its association with energy metabolism. Obes Rev. 2012;13:565-77. This paper reviews the evidence linking insufficient sleep with energy expenditure.

75. Hursel R, Rutters F, Gonnissen HK, et al. Effects of sleep fragmentation in healthy men on energy expenditure, substrate oxidation, physical activity, and exhaustion measured over $48 \mathrm{~h}$ in a respiratory chamber. Am J Clin Nutr. 2011;94:804-8.

76. Jung CM, Melanson EL, Frydendall EJ, et al. Energy expenditure during sleep, sleep deprivation and sleep following sleep deprivation in adult humans. J Physiol. 2011;589:235-44.

77. Benedict C, Hallschmid M, Lassen A, et al. Acute sleep deprivation reduces energy expenditure in healthy men. Am J Clin Nutr. 2011;93:1229-36.

78. • Nedeltcheva AV, Kilkus JM, Imperial J, et al. Insufficient sleep undermines dietary efforts to reduce adiposity. Ann Intern Med. 2010;153:435-41. This study showed for the first time that sleep restriction may attenuate the effects of caloric restriction.

79. Koban M, Swinson KL. Chronic REM-sleep deprivation of rats elevates metabolic rate and increases UCP1 gene expression in brown adipose tissue. Am J Physiol Endocrinol Metab. 2005;289: E68-74.

80. Cirelli C, Tononi G. Uncoupling proteins and sleep deprivation. Arch Ital Biol. 2004;142:541-9.

81. Chaput JP, Doyon CY, McNeil J, et al. Insufficient sleep is not associated with a greater adaptive reduction in thermogenesis during weight loss in adults. Bioenerg Open Access. 2012;1:104-8. 
82. - Cizza G, Marincola P, Mattingly M, et al. Treatment of obesity with extension of sleep duration: a randomized, prospective, controlled trial. Clin Trials. 2010;7:274-85. This proof-of-concept study on a randomized sample assesses whether sleep extension is feasible and whether it influences body weight.

83. - Chaput JP, Després JP, Bouchard C, Tremblay A. Longer sleep duration associates with lower adiposity gain in adult short sleepers. Int J Obes (Lond). 2012;36:752-6. This study showed for the first time that shifting sleep duration from a short to a healthier length is associated with an attenuation of fat mass gain in adults.

84. Rodríguez A, Gómez-Ambrosi J, Catalán V, et al. Acylated and desacyl ghrelin stimulate lipid accumulation in human visceral adipocytes. Int J Obes (Lond). 2009;33:541-52.

85. Tschöp M, Smiley DL, Heiman ML. Ghrelin induces adiposity in rodents. Nature. 2000;407:908-13.

86. - Chaput JP, Tremblay A. Sleeping habits predict the magnitude of fat loss in adults exposed to moderate caloric restriction. Obes Facts. 2012;5:561-6. This study provides evidence that sleeping habits can influence the success of a weight-loss intervention and should be taken into consideration when one decides to start a diet.

87. Elder CR, Gullion CM, Funk KL, et al. Impact of sleep, screen time, depression and stress on weight change in the intensive weight loss phase of the LIFE study. Int J Obes (Lond). 2012;36:86-92.

88. Hamilton WD. The genetical evolution of human behaviour. J Theor Biol. 1964; 7:1-52.

89. Siervo M, Wells CK, Cizza G. The contribution of psychological stress to the obesity epidemic: an evolutionary approach. Horm Metab Res. 2009;41:261-70.

90. Späth-Schwalbe E, Schöller T, Kern W, et al. Nocturnal adrenocorticotropin and cortisol secretion depends on sleep duration and decreases in association with spontaneous awakening in the morning. J Clin Endocrinol Metab. 1992;75:1431-5.

91. Kumari M, Badrick E, Ferrie J, et al. Self-reported sleep duration and sleep disturbance are independently associated with cortisol secretion in the Whitehall II study. J Clin Endocrinol Metab. 2009;94:4801-9. 